\title{
Robotic inguinal lymphadenectomy for penile cancer: the why, how, and what
}

\author{
Sammy E. Elsamra ${ }^{1}$, Michael A. Poch ${ }^{2}$ \\ ${ }^{1}$ Rutgers Robert Wood Johnson Medical School \& Rutgers Cancer Institute of New Jersey, New Brunswick, NJ, USA; ${ }^{2}$ Department of Genitourinary \\ Oncology, Moffitt Cancer Center, Tampa, FL, USA \\ Contributions: (I) Conception and design: All authors; (II) Administrative support: All authors; (III) Provision of study material or patients: None; \\ (IV) Collection and assembly of data: SE Elsamra; (V) Data analysis and interpretation: All authors; (VI) Manuscript writing: All authors; (VII) Final \\ approval of manuscript: All authors. \\ Correspondence to: Sammy E. Elsamra, MD. Department of Surgery (Urology), One Robert Wood Johnson Place, Division of Urology, Medical \\ Education Building, MEB 588-D, New Brunswick, NJ 08901, USA. Email: sammy.elsamra@rutgers.edu.
}

\begin{abstract}
Squamous cell carcinoma of the penis (SCC-P) demonstrates a reliable pattern of spread to the lymph nodes of the groin. High grade and higher stage (pT1b or greater) SCC-P demonstrate a higher propensity for metastasis to the inguinal lymph nodes. Further, lymphadenopathy progresses in a predictable fashion, from superficial inguinal lymph nodes to deep inguinal lymph nodes to pelvic lymph nodes, with limited survival noted for those patients with progression to pelvic lymph nodes. Fortunately, inguinal lymphadenectomy can provide cure and improvement in RFS for SCC-P. Unfortunately open inguinal lymphadenectomy is associated with significant morbidity. Perhaps owing to this morbidity, inguinal lymphadenectomy is underperformed in the US. In other words, urologists only offer inguinal lymphadenectomy for high risk SCC-P in only a minority of cases and even when performed, lymph node yield is often unsatisfactory (less than 8 nodes per groin). Recently, a laparoendoscopic inguinal lymphadenectomy has been developed as a new approach to offer potentially curative lymph node resection while minimizing morbidity. The robotic platform has since been adapted for this approach and several reports demonstrate significant improvements in morbidity while maintaining oncologic equivalency. This review highlights the rationale for inguinal lymphadenectomy, inguinal lymph node anatomy, and technical considerations and outcomes of laparoscopic and robotic inguinal lymphadenectomy.
\end{abstract}

Keywords: Inguinal lymphadenectomy; penile cancer; endoscopic subcutaneous modified inguinal lymphadenectomy (ESMIL); laparo-endoscopic groin (LEG) dissection; groin dissection; video-endoscopic inguinal lymphadenectomy (VEIL)

Submitted Apr 04, 2017. Accepted for publication May 18, 2017.

doi: 10.21037/tau.2017.06.05

View this article at: http://dx.doi.org/10.21037/tau.2017.06.05

\section{Introduction}

Squamous cell carcinoma of the penis (SCC-P) is rare malignancy in the United States, with an estimated incidence of less than 2,000 cases per year and less than 300 mortalities per year (Siegel 2016). Critical to the evaluation and management of SCC-P, however, is clear assessment of the inguinal lymph nodes for potential involvement. This is performed with a physical examination, in cases with difficult examination imaging evaluation. Standard care for inguinal involvement of regional lymph nodes is surgical excision when feasible (1-3). While not all palpable or enlarged lymph nodes are consistent with pathologic SCC-P nodal involvement (as some can be due to local infection and inflammation), delay in removal may result in progression to pelvic lymphadenopathy and non-regional metastatic disease which is associated with poor survival (4). Further not all regional inguinal metastases are palpable and inguinal lymph dissection (ILND) his has been shown to 
be of clinical in the prophylactic setting where microscopic metastatic disease is possible $(5,6)$.

Unfortunately open inguinal lymphadenectomy has been associated with significant morbidity with multiple modifications over the past 3 decades to limit complication risks (7).

This introduces a significant dilemma regarding the management of the groin in SCC-P. To limit open ILND to only those patients with palpable inguinal lymphadenopathy would expose many patients with occult metastasis to risk of spread and potentially worse oncologic outcomes while to perform open ILND on all patients with SCC-P would expose many patients to unnecessary morbidity.

Several strategies have been employed to address this clinical dilemma and they fall into three schemes: (I) utilizing information from the index tumor to help provide risk stratification; (II) improving detection of metastasis in regional lymph nodes; and (III) decreasing surgical morbidity of ILND.

It has become well recognized that certain features of the index tumor portend higher risk for metastasis to inguinal lymph nodes. Certain sub-types of SCC-P are more likely to metastasize (e.g., grossly ulcerative $v s$. nodular tumors and basaloid and sarcomatoid sub-types as opposed to verrucous or condylomatous histology type). The pathologic stage and grade of the index lesion offer significant information that suggests a risk of inguinal lymph node metastasis. High grade tumors (grade 3 and 4) and those with invasion into the corpora (T2) are at high risk for regional spread. T1b (invasion into lamina propria with LVI) tumors are also considered high risk for regional spread. Further, specific features seen on histology such as lympho-vascular invasion, peri-neural invasion, or a positive microscopic front are all associated with a higher risk for lymph node metastasis. In patients with clinically negative inguinal exam, nearly $0 \%$ of patients with $\mathrm{p}$ Tis and verrucous carcinoma will harbor micro-metastatic disease and $10 \%$ of patients with pTa and pT1a G1 penile tumors with harbor occult inguinal lymphadenopathy. Further, several series have identified an approximately $50 \%$ (38-90\%) occult regional metastasis rate in those with T2 disease. A nomogram developed by Ficarra $e t a l$. identified significant factors for lymph node involvement including: tumor thickness, growth pattern, grade, embolization group, corpora cavernosa infiltration, corpora spongiosum infiltration, and the presence of palpable lymph nodes (8).

Unfortunately, there are no imaging modalities that can reliably identify occult regional metastasis. Computerized tomography (CT), magnetic resonance imaging (MRI), and positron emission tomography (PET) have been utilized and certain features such as size of node or central necrosis or elevated SUV can suggest malignancy. However, they often fail to reliably predict presence of metastatic disease in patients without palpable lymphadenopathy $(\mathrm{CW})$.

Using radio-colloid and injectable dye, via a dynamic sentinel lymph node biopsy (DSLNB), may offer a better method to detect micro-metastatic disease. Several series have been published demonstrating the proficiency of DSLNB. However such expertise is often limited to highly specialized centers of excellence. A recent series describing outcomes DSLNB in 500 inguinal basins demonstrated only $92 \%$ sensitivity for DSLNB alone which improved to $95 \%$ with the addition of ultrasonic evaluation and fineneedle aspiration cytology (9). Hence, this demonstrates an excellent approach for patients with access to centers with such expertise. For patients far removed from such centers, perhaps, the most feasible strategy incorporates appropriate risk stratification for occult regional metastasis and minimization of morbidity associated with ILND. In addition to DSLNB, the use of percutaneous biopsy of palpable inguinal lymph nodes has been incorporated into management strategies (4).

Open ILND has been the recognized gold standard for the treatment of palpable inguinal lymphadenopathy (mobile or fixed) for decades. The technique entails creating a large incision, often in a hockey-stick fashion over the groin to allow for sufficient exposure of the saphenofemoral junction, several centimeters inferior to it, and $2 \mathrm{~cm}$ cephalad to the inguinal ligament. Often the saphenous vein is ligated at its insertion into the femoral vein. Clearly, such a large incision combined with removal of subcutaneous tissues rich in blood supply to the skin can place the patient at risk for devascularization of the skin flap created and result in significant wound infections. Further, the creation of a potential space and the propensity for lymphatic fluid to drain into and collect into this potential space creates significant risk for a seroma or lymphatic fluid collection that is ripe for infection. Further still, the skeletonization of the femoral vessels within the center of this compromised bed can lead to disastrous vascular complications. In fact, a sartorious flap, whereby the attachement of the sartorious muscle to the anterior superior iliac spine (ASIS) is detached and then re-attached medially on the inguinal ligament to cover the exposed major vessels of the thigh, is often necessary.

Recently, the use of a modified template to limit the 


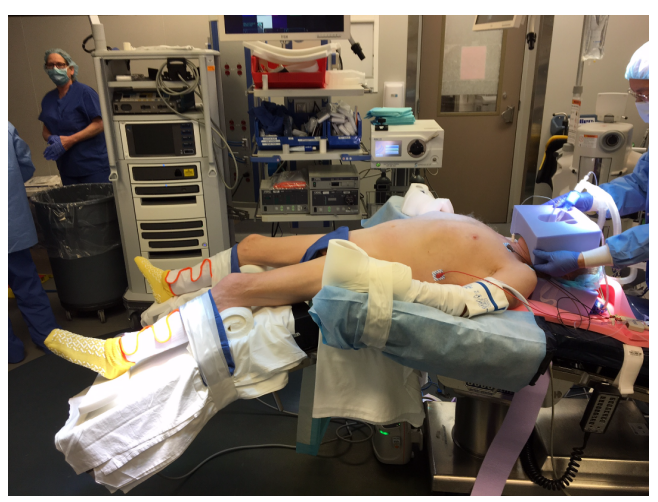

Figure 1 Patient positioning.

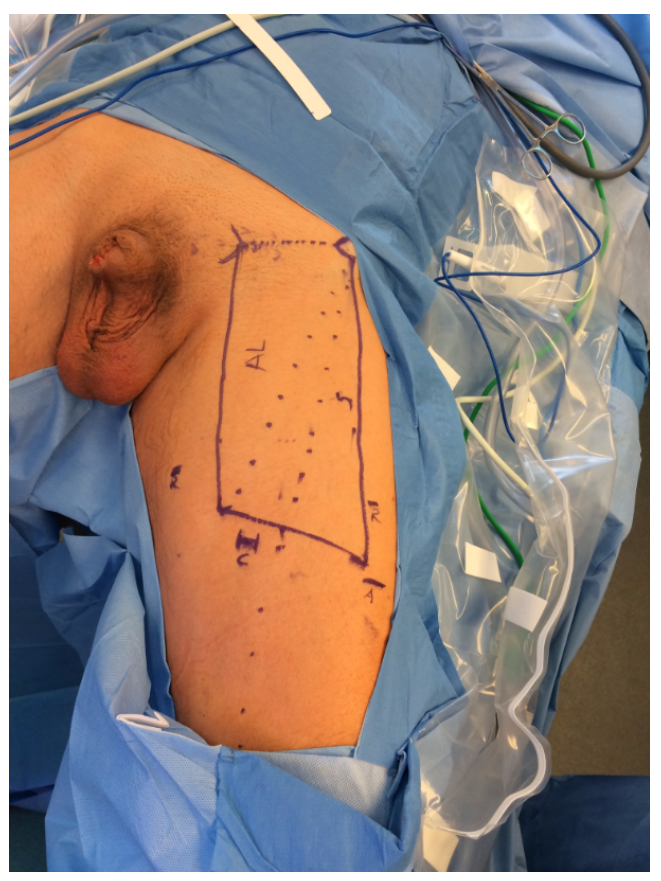

Figure 2 Port placement marking.

morbidity associated with full template open ILND (10-12). By utilizing a smaller incision (without the counterincision), sparing the saphenous vein, limiting the area of dissection to superior-medial to the sapheno-femoral junction, and not performing a sartorious flap, many of the risks for wound complications are mitigated. Even with such recent advancements in surgical technique, however, complications still occur in over $50 \%$ of patients undergoing open ILND (over a third of these were major complications); as described by a large series from four high volume centers (7). This series comprised of both full template standard and modified template inguinal lymphadenectomy. Another recent contemporary series of only modified template ILND (cases from 2002 to 2011) cited a lower complication rate of $10 \%$ with only a quarter of these complications being major (11). The concern persists, however, that one must not sacrifice lymph node yield for morbidity as many studies have demonstrated that greater lymph node yield and lower lymph node density portend survival benefit $(13,14)$.

\section{Laparoendoscopic approach to groin without or with the robot}

Initial experiences with laparoscopic ILND for groin were described for melanoma (15). This was then adopted by urologist for management of penile tumors. By hybridizing the techniques of subcutaneous eyebrow lift, saphenous vein harvesting, and laparoscopic surgery, Bishoff et al. provided the initial description of the laparoendoscopic approach to ILND [Bishoff JT, Basler JW, Teichman JM, et al. Endoscopic subcutaneous modified inguinal lymph node dissection (ESMIL) for squamous cell carcinoma of the penis. J Urol 2003.]. This dissection was initially conducted on cadaveric specimens and then applied to a patient with T3, N1, M0 SCC-P with success. The description of the laparoscopic approach is as follows:

(I) The patient is positioned with ipsilateral hip abducted and externally rotated. The thigh is well padded and secured and the skin is prepped from the umbilicus to the knee. Foley catheter is inserted (Figure 1);

(II) The borders of dissection are drawn out on the thigh: superior line drawn from ASIS to pubic tubercule, lateral line $20 \mathrm{~cm}$ inferiorly, medial line $15 \mathrm{~cm}$ inferiorly, inferior line connecting these who lateral lines (Figure 2);

(III) Port sites are marked (see image) and subcutaneous space is created using blunt and sharp dissection just under the dermis to allow for insertion of Hasson trocars for the medial and inferior ports. A $5-\mathrm{mm}$ port is placed lateral to the box (Figure 2);

(IV) Sub-dermal space is created using the surgeon's finger and/or lighted laparoscope to elevate a skin flap off of the underlying Camper's Fascia;

(V) Pneumoperitoneum is set to a level that prevents pneumo dissection; typically $8 \mathrm{mmHg}$; 
(VI) Early identification and preservation of the saphenous vein;

(VII) Dissection of lymph nodes from the four quadrants of the box, all superficial to the Fasica Lata, centered at the saphenofemoral junction;

(VIII) If deep ILND is necessary, the Fascia Lata is incised medial to the femoral vein and lymph tissue is extracted within the triangle formed by the inguinal ligament, the adductor longus muscle, and the sartorious muscle. The node of Cloquet is the most cephalad node within this deep packet. The femoral nerve is lateral to the femoral artery and dissection lateral to the artery should be avoided to prevent risk of nerve injury;

(IX) If the overlying skin is significantly compromised over the vessels (via injury or limited vascularity) a sartorious flap should be performed by dismembering the sartorious at its attachment with the ASIS and transposition over the vessels and securing its tendon to the inguinal ligament medially with 2-O PDS suture.

\section{Clinical experience with laparoscopic ILND}

Laparoendoscopic ILND has been performed by several groups since its initial description in 2003. It has also acquired several interesting names including Endoscopic Subcutaneous Modified ILND (ESMIL), video-endoscopic inguinal lymphadenectomy (VEIL), and laparo-endoscopic groin dissection (LEG). Most are small series demonstrating adequate lymph node yield with low complication rates and acceptable operative times and hospital length of stay. One series performed VEIL on one thigh while performing open on the other in 10 patients. Lymph node yields were similar, but complications were noticeably less and 9 of 10 patients preferred the VEIL (one patient did not prefer either surgery) (16). Cui et al. evaluated the benefit of saphenous vein sparing, in a side-by-side comparison (17). There was clear benefit in sparing the saphenous vein with decreased edema without decrease in lymph node yield. Kumar presented a case control series comparing 33 thighs dissected laparoendoscopically $v s .35$ thighs open (18). While all baseline characteristics were similar, lymph node yield was greater for VEIL despite having a higher number of positive lymph nodes. Further total wound complications were $6 \%$ for the VEIL compared to $68 \%$ for open. Most recently, the feasibility of single site approach to VEIL has been demonstrated (19). Table 1 delineates a summary of these studies.

\section{Robotic technique}

The laparoendoscopic technique described above has been applied to the robotic platform recently with success. Josephson et al. described the first such application in 2009. Since then there have been only a handful of case reports published, all demonstrating adequate lymph node yield with limited morbidity (20-23). The largest series published consists of a prospective Phase 1 study by Matin et al. whereby 20 thighs were dissected robotically. One thigh procedure was converted to open due to technical difficulty during the case (22). The remaining thighs were completed robotically and then a separate surgeon performed an open incision to evaluate for remaining lymph nodes. Eighteen of 19 thighs were adequately resected utilizing the robotic approach (see Table 1).

The authors (SEE) have experience with both laparoscopic and robotic approaches to ILND and favor the latter due to many factors. While our experience was initially with the laparoscopic approach, we found that in a bilateral dissection, the surgeon's position (always medial or lateral to all laparoscopic ports) resulted in poor ergonomics and resulted in significant strain. Also the benefit of articulating instruments afforded by the robotic platform allowed for greater facility in fine dissection. This potentially allowed for greater amount of preservation of venous tributaries; the superficial circumflex, superior epigastric, superficial external pudendal, and lateral accessory saphenous veins. Also, we have modified the technique by creating the working space between the fascia lata and the Scarpa's fascia. We have found this to be reliably created by dissecting our initial port incision down to the fascia lata directly and then creating a flap underneath Scarpa's fascia and expending it by utilizing a pre-peritoneal balloon dilator. This essentially allows for the superficial packet to be anterior to the working space, unlike descriptions for laparoscopic and robotic ILND published previously (23). With the da Vinci Xi platform, the 30 degree scope, which can be rotated up and down by the surgeon at the console, allows for facile dissection both anteriorly (superficial packet) and posteriorly (deep packet). The rotating boom on the da Vinci Xi platform also allows for side-docking (see Figures 3,4).

At our institution (Rutgers Cancer Institute of New Jersey), expertise with DSLNB is not readily available, and hence we have adopted the strategy of combining 
Table 1 Published clinical series* with laparoscopic and robotic inguinal lymphadenectomy

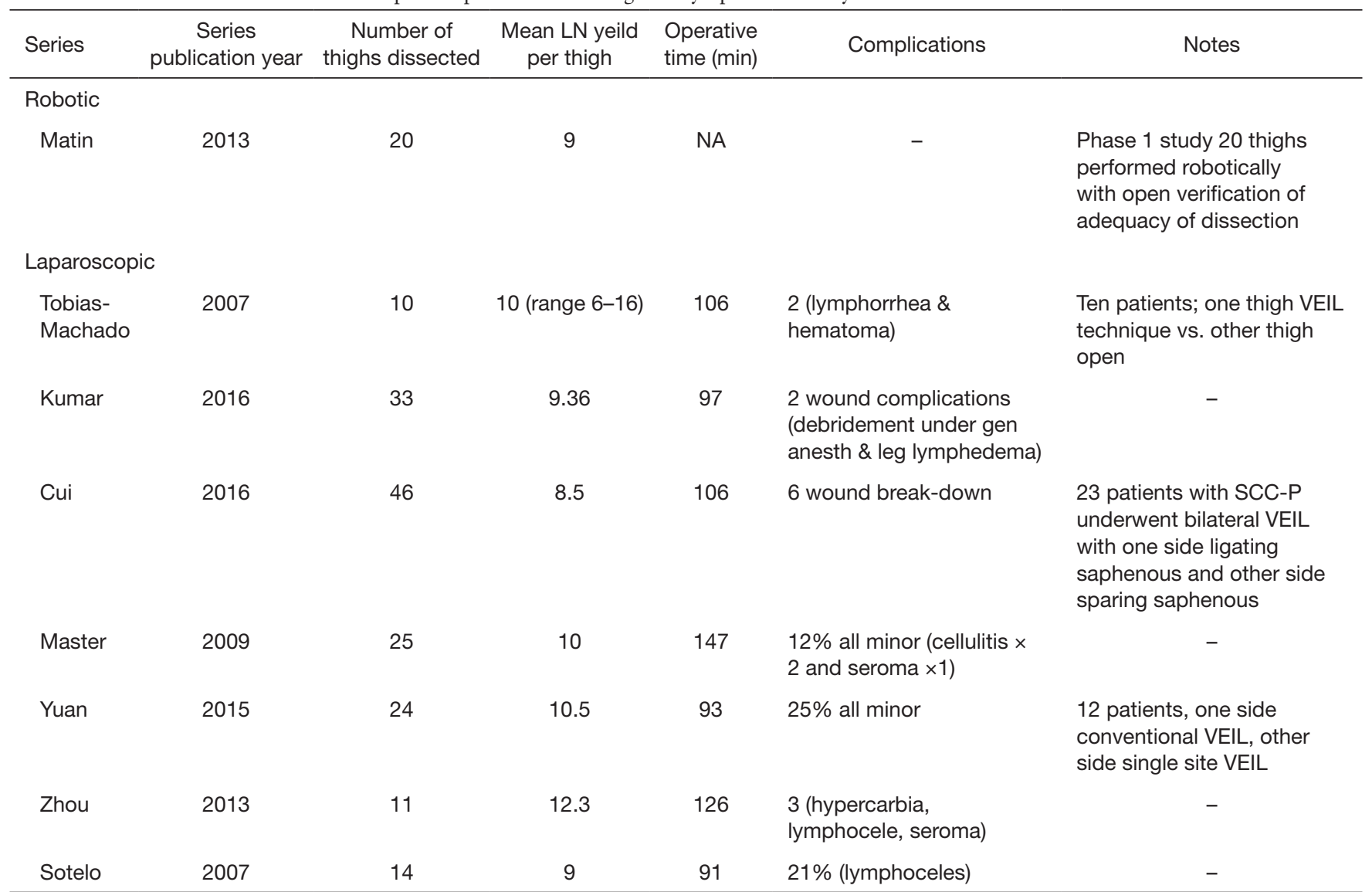

*, excludes published case reports and non-PubMed cited series. NA, not available; VEIL, video-endoscopic inguinal lymphadenectomy.
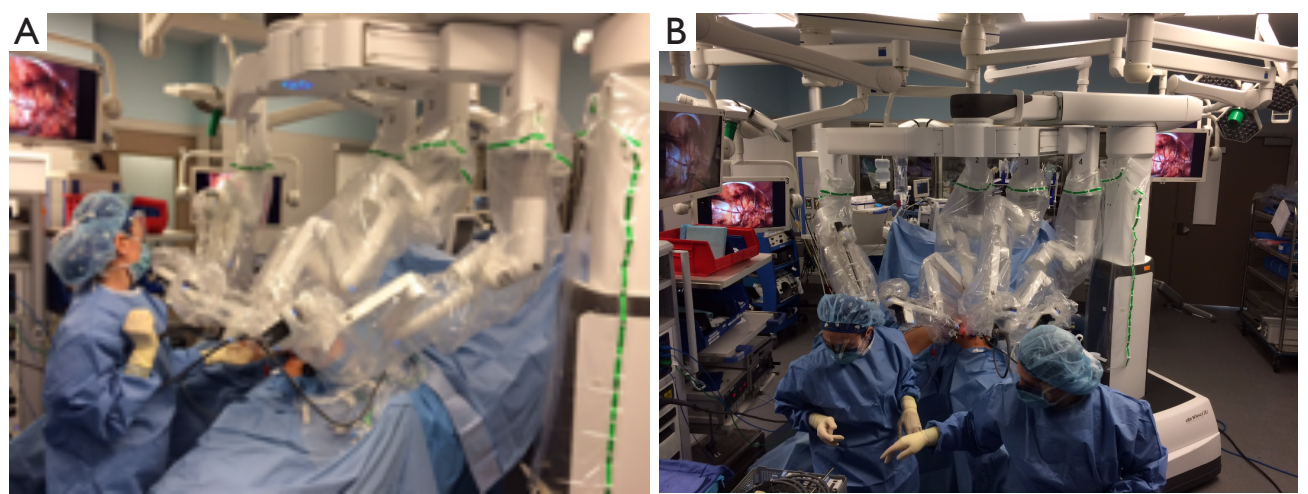

Figure 3 Robotic docking.

appropriate risk stratification and minimizing morbidity associated with ILND by offering anyone with pT1b or greater SCC-P a bilateral robotic ILND. We have also applied this technique to patients with truncal and extremity melanoma at the request of our surgical oncology colleagues. At the time of this manuscript with have performed robotic ILND on 11 thighs and have had no major complications. One patient developed epidermolysis which healed with a damp to dry dressing. One patient has developed right lower extremity neuropathic pain and is under the care of pain management for this. All our patients have had only an overnight hospital stay. Our mean 


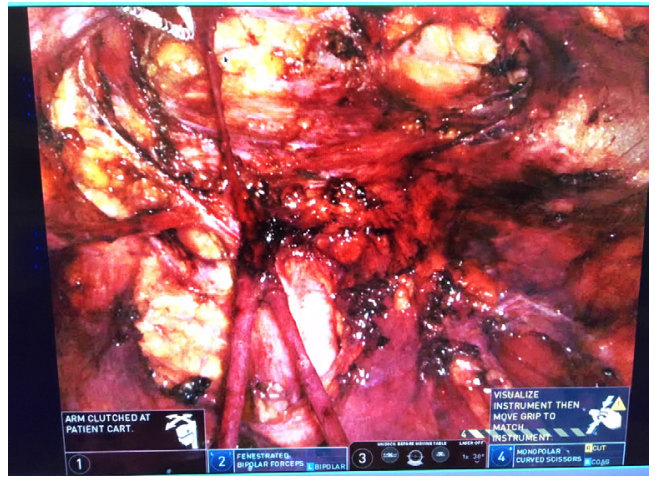

Figure 4 Still image of robotic view of completed right superficial inguinal lymphadenectomy.

Table 2 Summary of clinical experience of RILND in 10 thighs at Rutgers Cancer Institute of New Jersey

\begin{tabular}{lc}
\hline Operative and perioperative parameters & Mean result (range) \\
\hline Procedure time (per limb, min) & $151.4(99.0-224.0)$ \\
Estimated blood loss $(\mathrm{mL})$ & $30(12.5-50.0)$ \\
Lymph node yeild per limb & $10.4(6.0-16.0)$ \\
Length of stay (days) & $1(1.0)$ \\
Number of complications & - \\
Minor (Clavien 1-2) & 5 \\
Major (Clavien 3a or greater) & 1 \\
\hline
\end{tabular}

operative time per thigh is $151 \mathrm{~min}$ with improvement as case volume increased. Our lymph node yield also improved with increasing case number ranging from 3 to 18; with 3 lymph nodes harvested on our first case (done laparoscopically) and 18 harvested in our $9^{\text {th }}$ thigh dissected (performed robotically). Our experience demonstrated that appropriate lymph node yields of 7 or greater were achieved by our third thigh dissection (refer to Table 2).

Our early experience included one patient with a single mobile lymph node. Fortunately this was our $9^{\text {th }}$ thigh dissection. We noted that much of the accessory vein sparing feasible in the earlier cases was not feasible during this case as they were fixed with the involved lymph node. Robotic ILND is perhaps best suited for prophylactic ILND or at most for therapeutic lymphadenopathy with no greater than $\mathrm{cN} 1$, preferably after the learning curve is cleared. Fixed lymphadenopathy may obscure planes greatly and require skin resection obfuscating the benefit of robotic ILND; certainly these cases should only be attempted on a case-by-case basis by highly experienced surgeons with much patient counseling provided.

\section{Conclusions}

Laparoscopic and Robotic ILND for prophylactic and early therapeutic purposes is feasible and associated with adequate lymph node yield and decreased morbidity in experienced hands. Proper patient selection based on index tumor properties is critical to identify those patients who are at high risk for occult regional spread and hence those who would likely benefit from a prophylactic ILND. A more favorable morbidity profile may decrease the burden of complications on those undergoing prophylactic ILND. While many series have been published demonstrating excellent outcomes, these are generally from centers with high volume and one must appreciate the learning curve associated with minimally invasive inguinal lymphadenectomies.

\section{Acknowledgements}

None.

\section{Footnote}

Conflicts of Interest: Dr. Elsamra is surgical proctor for intuitive surgical; Dr. Poch has no conflicts of interest to declare.

\section{References}

1. Protzel C, Alcaraz A, Horenblas S, et al. Lymphadenectomy in the surgical management of penile cancer. Eur Urol 2009;55:1075-88.

2. Heyns CF, Fleshner N, Sangar V, et al. Management of the lymph nodes in penile cancer. Urology 2010;76:S43-57.

3. Marconnet L, Rigaud J, Bouchot O. Long-term followup of penile carcinoma with high risk for lymph node invasion treated with inguinal lymphadenectomy. J Urol 2010;183:2227-32.

4. Djajadiningrat RS, Graafland NM, van Werkhoven E, et al. Contemporary management of regional nodes in penile cancer-improvement of survival? J Urol 2014;191:68-73.

5. Kroon BK, Horenblas S, Lont AP, et al. Patients with penile carcinoma benefit from immediate resection of clinically occult lymph node metastases. J Urol 2005;173:816-9. 
6. McDougal WS. Preemptive lymphadenectomy markedly improves survival in patients with cancer of the penis who harbor occult metastases. J Urol 2005;173:681.

7. Gopman JM, Djajadiningrat RS, Baumgarten AS, et al. Predicting postoperative complications of inguinal lymph node dissection for penile cancer in an international multicentre cohort. BJU Int 2015;116:196-201.

8. Ficarra V, Zattoni F, Artibani W, et al. Penile Cancer Project Members.. Nomogram predictive of pathological inguinal lymph node involvement in patients with squamous cell carcinoma of the penis. J Urol 2006;175:1700-4; discussion 1704-5.

9. Lam W, Alnajjar HM, La-Touche S, et al. Dynamic sentinel lymph node biopsy in patients with invasive squamous cell carcinoma of the penis: a prospective study of the long-term outcome of 500 inguinal basins assessed at a single institution. Eur Urol 2013;63:657-63.

10. Catalona WJ. Modified inguinal lymphadenectomy for carcinoma of the penis with preservation of saphenous veins: technique and preliminary results. J Urol 1988;140:306-10.

11. Koifman L, Hampl D, Koifman N, et al. Radical open inguinal lymphadenectomy for penile carcinoma: surgical technique, early complications and late outcomes. J Urol 2013;190:2086-92.

12. Tsaur I, Biegel C, Gust K, et al. Feasibility, complications and oncologic results of a limited inguinal lymph node dissection in the management of penile cancer. Int Braz J Urol 2015;41:486-95.

13. Thuret R, Sun M, Lughezzani G, et al. A contemporary population-based assessment of the rate of lymph node dissection for penile carcinoma. Ann Surg Oncol 2011;18:439-46.

14. Li ZS, Deng CZ, Yao K, et al. Bilateral pelvic lymph node dissection for Chinese patients with penile cancer: a multicenter collaboration study. J Cancer Res Clin Oncol

Cite this article as: Elsamra SE, Poch MA. Robotic inguinal lymphadenectomy for penile cancer: the why, how, and what. Transl Androl Urol 2017;6(5):826-832. doi: 10.21037/ tau.2017.06.05
2017;143:329-35.

15. Trias M, Targarona EM, Piulachs J, et al. Extraperitoneal laparoscopically assisted ilioinguinal lymphadenectomy for treatment of malignant melanoma. Arch Surg 1998;133:272-4; discussion 275.

16. Tobias-Machado M, Tavares A, Ornellas AA, et al. Video endoscopic inguinal lymphadenectomy: a new minimally invasive procedure for radical management of inguinal nodes in patients with penile squamous cell carcinoma. $\mathrm{J}$ Urol 2007;177:953-7; discussion 958.

17. Cui Y, Chen H, Liu L, et al. Saphenous vein sparing during laparoscopic bilateral inguinal lymphadenectomy for penile carcinoma patients. Int Urol Nephrol 2016;48:363-6.

18. Kumar V, Sethia KK. Prospective study comparing video-endoscopic radical inguinal lymph node dissection (VEILND) with open radical ILND (OILND) for penile cancer over an 8-year period. BJU Int 2017;119:530-4.

19. Tobias-Machado M, Correa WF, Reis LO, et al. Singlesite video endoscopic inguinal lymphadenectomy: initial report. J Endourol 2011;25:607-10.

20. Josephson DY, Jacobsohn KM, Link BA, et al. Roboticassisted endoscopic inguinal lymphadenectomy. Urology 2009;73:167-70; discussion 170-1.

21. Dogra PN, Saini AK, Singh P. Robotic-assisted inguinal lymph node dissection: A preliminary report. Indian J Urol 2011;27:424-7.

22. Matin SF, Cormier JN, Ward JF, et al. Phase 1 prospective evaluation of the oncological adequacy of robotic assisted video-endoscopic inguinal lymphadenectomy in patients with penile carcinoma. BJU Int 2013;111:1068-74.

23. Sotelo R, Cabrera M, Carmona O, et al. Robotic bilateral inguinal lymphadenectomy in penile cancer, development of a technique without robot repositioning: a case report. Ecancermedicalscience 2013;7:356. 